\title{
Covid-19 e tuberculose: coinfecção e riscos
}

\author{
Covid-19 and tuberculosis: coinfection and risks
}

Covid-19 y tuberculosis: coinfección y riesgos

\author{
Mellyne Henriques Guerra \\ ORCID: https://orcid.org/0000-0002-0578-281X \\ Universidade Tiradentes, Brasil \\ E-mail: mellynehenriques@gmail.com \\ Ana Caroline Gusmão de Matos \\ ORCID: https://orcid.org/0000-0002-4451-0243 \\ Universidade Tiradentes, Brasil \\ E-mail: anagusmaao@gmail.com \\ José Bento dos Santos \\ ORCID: https://orcid.org/0000-0001-8190-325X \\ Universidade Federal de Sergipe, Brasil \\ E-mail: jbento17@hotmail.com \\ Déborah Esteves Carvalho \\ ORCID: https://orcid.org/0000-0001-8071-0069 \\ Universidade Tiradentes, Brasil \\ E-mail: deborah.escv@gmail.com \\ Manuelli Antunes da Silva \\ ORCID: https://orcid.org/0000-0002-7776-1196 \\ Universidade Tiradentes, Brasil \\ E-mail: manuellibrito@hotmail.com \\ Tatiana Martins Araújo Ribeiro \\ ORCID: https://orcid.org/0000-0001-8178-473X \\ Universidade Tiradentes, Brasil \\ E-mail: ribeirotatianama@gmail.com \\ Carlos Alberto Góis Barreto \\ ORCID: https://orcid.org/0000-0003-1319-4919 \\ Universidade Federal de Sergipe, Brasil \\ E-mail: carlosagbarreto@outlook.com \\ Cristóvão Almeida Barros \\ ORCID: https://orcid.org/0000-0002-0617-9166 \\ Universidade Tiradentes, Brasil \\ E-mail: cristovao02@hotmail.com \\ Filipe de Almeida Barbosa \\ ORCID: https://orcid.org/0000-0003-2644-4040 \\ Universidade Tiradentes, Brasil \\ E-mail: filipe-lipi@hotmail.com \\ Carla Viviane Freitas de Jesus \\ ORCID: https://orcid.org/0000-0002-7775-6610 \\ Universidade Tiradentes, Brasil \\ E-mail: carlavfj@gmail.com
}

\begin{abstract}
Resumo
Objetivo: Avaliar as características, evolução e desfecho dos pacientes coinfectados por COVID-19 e tuberculose (TB). Metodologia: A fim de responder a pergunta norteadora "Quais os riscos da coinfecção por COVID-19 e tuberculose?", foi realizada uma revisão integrativa da literatura, qualitativa, com abordagem descritiva, nas bases de dados Google Scholar, PubMed, Cochrane Library, SciELO e LILACS, utilizando os descritores "COVID-19", "Tuberculosis" e "Co-infection". Foram critérios de inclusão: artigo disponível na íntegra, publicado em 2020, nos idiomas português, inglês e/ou espanhol. De exclusão artigos sem relação com o tema, com foco em ferramentas estatísticas, resumos isolados, capítulos de livros, editoriais, artigos de revisão, fichas técnicas, teses, dissertações e trabalhos de conclusão de curso. Resultados: Foram incluídos 8 artigos. A maioria dos pacientes coinfectados foram do sexo masculino, com média de idade de 47,19 anos. Os sintomas mais relatados foram febre, tosse, dispneia e cefaleia. As principais alterações laboratoriais incluíram elevação de proteína $\mathrm{C}$ reativa, velocidade de hemossedimentação, D-dímero e fibrinogênio. Achados imaginológicos como opacificações, derrame pleural, "árvore em brotamento", vidro fosco, cavitações, atelectasias e espessamento do septo lobar foram frequentes. A coinfecção esteve mais atrelada a casos severos da doença e a letalidade foi de até 33,3\%. Socialmente, a coinfecção repercute em
\end{abstract}


dificuldades em diagnóstico diferencial, morbidade em grupos vulneráveis e dificuldade de acesso a tratamento em cenário pandêmico. Conclusão: Alguns estudos divergiram acerca da letalidade, porém foi possível observar uma tendência à associação de gravidade do curso clínico nos pacientes coinfectados.

Palavras-chave: Coinfecção; Infecções por coronavirus; Prognóstico; Tuberculose.

\begin{abstract}
Objective: To evaluate the characteristics, evolution and outcome of patients co-infected with COVID-19 and tuberculosis (TB). Methodology: In order to answer the guiding question "What are the risks of coinfection by COVID-19 and tuberculosis?", an integrative literature review, qualitative, with a descriptive approach, was carried out in the Google Scholar, PubMed, Cochrane Library, SciELO and LILACS databases, using the descriptors "COVID -19", "Tuberculosis" and "Co-infection". Inclusion criteria were: article available in full, published in 2020, in Portuguese, English and/or Spanish. Articles unrelated to the topic, with a focus on statistical tools, isolated abstracts, book chapters, editorials, review articles, technical sheets, theses, dissertations and course completion papers were excluded. Results: 8 articles were included. Most of the co-infected patients were male, with a mean age of 47.19 years. The most reported symptoms were fever, cough, dyspnoea and headache. The main laboratory changes included elevation of C-reactive protein, erythrocyte sedimentation rate, D-dimer and fibrinogen. Imagological findings such as opacifications, pleural effusion, "sprouting tree", ground glass, cavitations, atelectasis and thickening of the lobar septum were frequent. Co-infection was more linked to severe cases of the disease and lethality was up to $33.3 \%$. Socially, co-infection results in difficulties in differential diagnosis, morbidity in vulnerable groups and difficulty in accessing treatment in a pandemic scenario. Conclusion: Some studies differed on lethality, but it was possible to observe a trend towards an association between the severity of the clinical course in co-infected patients.
\end{abstract}

Keywords: Coinfection; Coronavirus infections; Prognosis; Tuberculosis.

\title{
Resumen
}

Objetivo: Evaluar las características, evolución y resultados de los pacientes coinfectados por COVID-19 y tuberculosis (TB). Metodología: Para dar respuesta a la pregunta orientadora “¿Cuáles son los riesgos de coinfección por COVID-19 y tuberculosis?", se realizó una revisión integradora de la literatura, cualitativa, con un enfoque descriptivo, en las bases de datos Google Scholar, PubMed, Cochrane Library, SciELO y LILACS, utilizando los descriptores "COVID -19", “Tuberculosis" y "Coinfección". Los criterios de inclusión fueron: artículo disponible completo, publicado en 2020, en portugués, inglés y/o español. Exclusión de artículos no relacionados con el tema, con enfoque en herramientas estadísticas, resúmenes aislados, capítulos de libros, editoriales, artículos de revisión, fichas técnicas, tesis, disertaciones y trabajos de finalización de cursos. Resultados: Se incluyeron 8 artículos. La mayoría de los pacientes coinfectados eran varones, con una edad media de 47,19 años. Los síntomas más notificados fueron fiebre, tos, disnea y dolor de cabeza. Los principales cambios de laboratorio incluyeron elevación de proteína C reactiva, velocidad de sedimentación globular, dímero D y fibrinógeno. Fueron frecuentes los hallazgos imagológicos como opacificaciones, derrame pleural, "árbol de brotación", vidrio esmerilado, cavitaciones, atelectasias y engrosamiento del septo lobular. La coinfección estuvo más relacionada con los casos graves de la enfermedad y la letalidad fue de hasta un 33,3\%. Socialmente, la coinfección genera dificultades en el diagnóstico diferencial, morbilidad en grupos vulnerables y dificultad para acceder al tratamiento en un escenario pandémico. Conclusión: Algunos estudios difirieron en la letalidad, pero fue posible observar una tendencia hacia una asociación entre la gravedad del curso clínico en pacientes coinfectados.

Palabras clave Coinfección; Infecciones por coronavirus; Pronóstico; Tuberculosis.

\section{Introdução}

A Organização Mundial da Saúde (OMS) declarou o surto do novo coronavírus (COVID-19) como uma emergência de saúde pública internacional em 30 de janeiro de 2020 (Sarinoglu et al., 2020). Dia 24 de março foi o Dia Mundial da Tuberculose e ao final desta semana, em 2020, a COVID-19 há havia ultrapassado 600 mil casos confirmados e cerca de 30 mil mortes no mundo (Adepoju, 2020).

A pandemia alterou a rotina de trabalho dos profissionais de saúde, bem como a logística do atendimento médico e hospitalar. No que diz respeito à tuberculose (TB), o fornecimento e transporte de medicamentos foram afetados por cancelamentos de voos e restrições de viagens impostas. A interrupção dos tratamentos é um fator preocupante, pois pode ocasionar resistência aos medicamentos, que é uma grande ameaça para o controle global, principalmente onde a epidemia é 
impulsionada pelo vírus da imunodeficiência humana (HIV) (Adepoju, 2020). Outrossim, o isolamento pode dificultar os serviços de prevenção e interromper os testes de campo.

Além das implicações sobre os serviços e os pacientes de tuberculose, surgem novas preocupações a serem investigadas como a possível associação entre TB e COVID-19, a reativação da TB em pacientes já tratados e os efeitos na mortalidade precoce dos pacientes infectados pelo SARS-CoV-2 (Tadolini et al., 2020b). É bem documentado que algumas infecções virais, como sarampo, agravam a tuberculose pulmonar por deprimirem a imunidade celular (He, 2020), o que pode também ocorrer com o SARS-CoV-2.

A similaridade entre os sinais e sintomas iniciais da COVID-19 e TB, como tosse, febre e dispneia, reforçam o desafio para discernir entre diagnóstico diferencial, coinfecção ou reativação da TB (Tadolini et al., 2020a). Tendo em vista que a tuberculose é a principal causa de morte no mundo por um único agente infeccioso (1,5 milhão de óbitos por ano) e o cenário atual de pandemia pelo SARS-CoV-2, o presente estudo tem como objetivo avaliar os aspectos diagnósticos e prognósticos de pacientes coinfectados com tuberculose e COVID-19.

\section{Metodologia}

Trata-se de uma revisão integrativa da literatura, qualitativo, com abordagem descritiva, a partir de estudos que discutem de maneira total ou parcial o tema proposto. Para sua execução, foram respeitadas as fases desse tipo de pesquisa: 1Elaboração da pergunta norteadora; 2- Busca ou amostragem na literatura; 3- Coleta de dados; 4- Análise crítica dos estudos incluídos; 5- Discussão dos resultados; 6- Apresentação da revisão integrativa (Souza, Silva \& Carvalho, 2010; Pereira et al, 2018; Koche, 2011; Ludkw \& André, 2013).

Para responder à pergunta norteadora “Quais os riscos da coinfecção por COVID-19 e tuberculose?”, foi realizado levantamento de artigos nas bases de dados Scientific Electronic Library Online (SciELO), PubMed, Cochrane Library, Literatura Latino-Americana e do Caribe em Ciências da Saúde (LILACS) e Google Scholar. Os descritores utilizados foram "COVID-19", "Tuberculosis" e "Co-infection"; utilizando o operador booleano AND. Como critérios de inclusão foram estabelecidos: artigo original, disponível na íntegra para livre acesso, publicado em 2020, nos idiomas português, inglês e/ou espanhol. Foram excluídos os artigos que não tinham relação com o objeto de estudo, os que focaram em ferramentas estatísticas para estimativas epidemiológicas, resumos isolados, capítulos de livros, editoriais, artigos de revisão, fichas técnicas, dissertações, teses e demais trabalhos de conclusão de curso.

Em seguida, foram avaliados os títulos e resumos a fim de identificar resultados de interesse para a revisão. Nos casos em que a leitura do resumo não foi suficiente para definir se o trabalho deveria ser incluído na amostra, foram considerados os demais critérios de inclusão e exclusão. Com vistas a determinar sua elegibilidade, os artigos selecionados foram lidos na íntegra. Os dados referentes à metodologia (desenho de estudo, local e ano de realização) empregada nos artigos que compuseram a amostra final foram sistematicamente selecionados a partir de quadro sinóptico elaborado pelos pesquisadores, o que facilitou a comparação e interpretação das informações (Quadro 1).

\section{Resultados}

A busca inicial nas bases de dados a partir dos descritores retornou 1.965 trabalhos, cuja maior parte foi obtida no Google Scholar (n=2.280), seguido de PubMed ( $\mathrm{n}=68)$, Cochrane Library ( $\mathrm{n}=2)$ e LILACS (n=2). A busca no SciELO não encontrou resultados. Desse total, 26 artigos foram selecionados para a leitura na íntegra, contudo, apenas 8 contemplaram os critérios de elegibilidade da revisão (Figura 1). 
Figura 1. Fluxograma do processo de seleção de estudos.

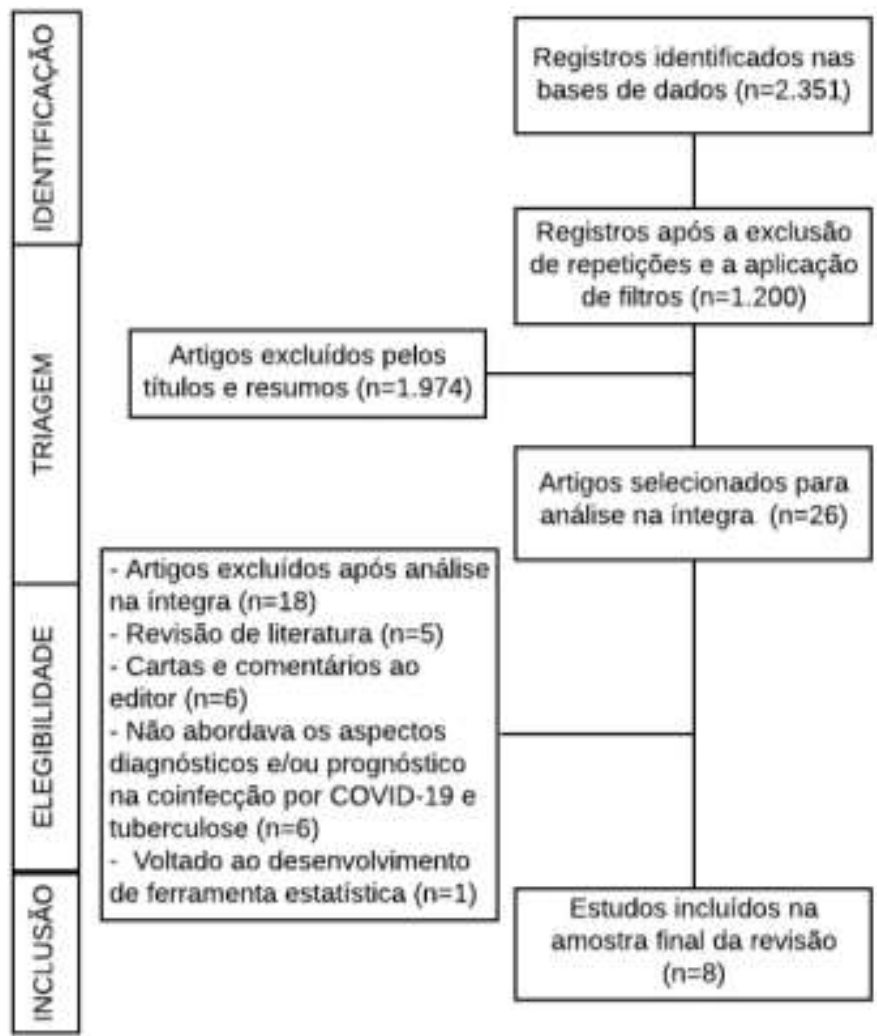

Fonte: Autores.

Um dos 8 artigos inclusos no estudo, utilizou a coleta de dados multicêntrica, realizada em 8 países. Turquia, Itália, Cingapura, China, Catar e Estados Unidos produziram, cada um, um dentre os artigos selecionados, os demais não especificaram o país de origem. Todas as pesquisas selecionadas foram referentes ao ano de 2020, uma vez que as publicações a respeito do SARS-CoV-2, identificado pela primeira vez apenas no final de dezembro, têm sofrido atualizações constantes. Quanto ao idioma, todos os oito estudos foram publicados na língua inglesa. Quanto aos tipos de pesquisa, um estudo foi definido como coorte e 8 como relato de caso, em que dois estudos foram retrospectivos.

Quadro 1. Distribuição dos estudos segundo autor, título, desenho, país da realização e ano da publicação.

\begin{tabular}{|l|l|l|l|}
\hline \multicolumn{1}{|c|}{ Autor/Ano } & \multicolumn{1}{|c|}{ Título } & Desenho do estudo & \multicolumn{1}{|c|}{ País / Ano } \\
\hline $\begin{array}{l}\text { THAM et al., } \\
\mathbf{2 0 2 0}\end{array}$ & $\begin{array}{l}\text { Four Patients with COVID-19 and } \\
\text { Tuberculosis, Singapore, April-May } \\
2020\end{array}$ & Estudo de caso. & Cingapura / 2020 \\
\hline $\begin{array}{l}\text { FREIJ et al., } \\
\mathbf{2 0 2 0}\end{array}$ & $\begin{array}{l}\text { Fatal central nervous system co- } \\
\text { infection with SARS-CoV-2 and } \\
\text { tuberculosis in a healthy child }\end{array}$ & Estudo de caso. & Não informado / 2020 \\
\hline $\begin{array}{l}\text { YAO et al., } \\
\mathbf{2 0 2 0}\end{array}$ & $\begin{array}{l}\text { Three Patients with COVID-19 and } \\
\text { Pulmonary Tuberculosis, Wuhan, }\end{array}$ & Estudo de caso. & China / 2020 \\
\hline
\end{tabular}


Research, Society and Development, v. 10, n. 2, e0710212257, 2021

(CC BY 4.0) | ISSN 2525-3409 | DOI: http://dx.doi.org/10.33448/rsd-v10i2.12257

\begin{tabular}{|c|c|c|c|}
\hline & China, January-February 2020 & & \\
\hline $\begin{array}{l}\text { YOUSAF et } \\
\text { al., } 2020\end{array}$ & $\begin{array}{l}\text { Cavitary pulmonary tuberculosis with } \\
\text { COVID-19 coinfection }\end{array}$ & $\begin{array}{l}\text { Estudo de caso } \\
\text { retrospectivo. }\end{array}$ & Catar / 2020 \\
\hline $\begin{array}{l}\text { LUCIANI et } \\
\text { al., } 2020\end{array}$ & $\begin{array}{l}\text { Coinfection of Tuberculosis } \\
\text { Pneumonia and COVID-19 in a Patient } \\
\text { Vaccinated with Bacille Calmette- } \\
\text { Guérin (BCG): Case Report }\end{array}$ & Estudo de caso. & Itália / 2020 \\
\hline $\begin{array}{l}\text { ATA et al., } \\
2020\end{array}$ & $\begin{array}{l}\text { A 28-Year-Old Man from India with } \\
\text { SARS-Cov-2 and Pulmonary } \\
\text { Tuberculosis Co-Infection with Central } \\
\text { Nervous System Involvement }\end{array}$ & Estudo de caso. & Não informado / 2020 \\
\hline $\begin{array}{l}\text { SARINOGLU } \\
\text { et al., } 2020\end{array}$ & $\begin{array}{l}\text { Tuberculosis and COVID-19: An } \\
\text { overlapping situation during pandemic }\end{array}$ & Estudo de caso. & Turquia / 2020 \\
\hline $\begin{array}{l}\text { MOTTA et } \\
\text { al., } 2020\end{array}$ & $\begin{array}{l}\text { Tuberculosis, COVID-19 and } \\
\text { migrants: Preliminary analysis of } \\
\text { deaths occurring in } 69 \text { patients from } \\
\text { two cohorts }\end{array}$ & $\begin{array}{l}\text { Estudo de coorte } \\
\text { retrospectivo } \\
\text { multicêntrico. }\end{array}$ & $\begin{array}{l}\text { Multicêntrico (Bélgica, } \\
\text { Brasil, França, Itália, Rússia, } \\
\text { Cingapura, Espanha e Suíça) } \\
\text { / } 2020\end{array}$ \\
\hline
\end{tabular}

Fonte: Autores.

Os principais resultados encontrados estão sintetizados no Quadro 2. Foram avaliados 26 pacientes, $80,8 \%$ do sexo masculino, com idade entre 5 e 82 anos, média de 47,19 anos. Os sinais e sintomas mais comumente referidos foram, em ordem de frequência nos estudos: febre (75\%), tosse $(62,5 \%)$, dispneia $(37,5 \%)$ e cefaleia $(37,5 \%)$.

Dentre os achados laboratoriais, destacam-se a marcante linfopenia, níveis elevados de proteína $\mathrm{C}$ reativa, velocidade de hemossedimentação aumentada, elevação de D-dímero e fibrinogênio. O exame utilizado para diagnosticar a infecção por SARS-CoV-2 em todos os estudos avaliados foi o RT-PCR de swab nasofaríngeo e o diagnóstico de TB foi principalmente realizado por esfregaço e/ou cultura do escarro.

Os achados imaginológicos mais frequentes foram opacificações difusas, derrame pleural, aspecto de "árvore em brotamento", vidro fosco, cavitações, atelectasias e espessamento do septo lobar. A TB foi tratada com drogas antituberculosas na grande maioria dos estudos, com destaque em especial o esquema terapêutico HRZE (isoniazida, rifampicina, pirazinamida e etambutol). Como abordagem terapêutica à COVID-19 foram utilizados antivirais, hidroxicloroquina e associação com antibioticoterapia. Oxigenioterapia se fez necessária em 6 pacientes avaliados.

A duração da hospitalização variou de 22 dias a 130 dias. Por fim, a letalidade variou entre $11,6 \%$ e 33,3\% nos estudos com amostra maior que um paciente, com exceção de Yousaf et al. (2020), em que não houve óbitos. 
Quadro 2. Principais resultados obtidos por estudo incluído conforme perfil demográfico, sintomas, exames, evolução e desfecho

\begin{tabular}{|c|c|c|c|c|c|c|c|c|}
\hline Autores & THAM et al., 2020 & $\begin{array}{l}\text { FREIJ et al., } \\
2020\end{array}$ & YAO et al., 2020 & YOUSAF et al., 2020 & $\begin{array}{l}\text { LUCIANI et al., } \\
2020\end{array}$ & $\begin{array}{l}\text { ATA et al., } \\
2020\end{array}$ & $\begin{array}{l}\text { SARINOGLU et al., } \\
2020\end{array}$ & MOTTA et al., 2020 \\
\hline $\begin{array}{l}\text { Número de } \\
\text { coinfectados }\end{array}$ & 4 pacientes & 1 paciente & 3 pacientes & 6 pacientes & 1 paciente & 1 paciente & 2 pacientes & 8 pacientes \\
\hline Sexo & Masculino - $100 \%$ & $\begin{array}{l}\text { Feminino }- \\
100 \%\end{array}$ & Masculino - $100 \%$ & Masculino - $100 \%$ & Feminino $-100 \%$ & $\begin{array}{l}\text { Masculino - } \\
100 \%\end{array}$ & Feminino $-100 \%$ & $\begin{array}{l}\text { Masculino - } 87,5 \% \\
\text { Feminino - } 12,5 \%\end{array}$ \\
\hline Idade & Entre 22 e 40 anos & 5 anos & 44 a 57 anos & 27 a 50 anos & 32 anos & 28 anos & 39 e 77 anos & Entre 45 e 82 anos \\
\hline Sinais e sintomas & $\begin{array}{l}\text { Febre }(100 \%), \text { tosse } \\
\text { produtiva }(50 \%), \text { tosse } \\
\text { não produtiva }(50 \%), \\
\text { perda de peso }(25 \%), \\
\text { dispneia aos esforços } \\
(25 \%), \text { dor torácica } \\
\text { pleurítica }(25 \%) \text {, baixa } \\
\text { tolerância aos esforços } \\
(25 \%) \text {. }\end{array}$ & Febre e cefaleia. & $\begin{array}{l}\text { Febre, tosse, fadiga, espirros e } \\
\text { perda de peso }(100 \%) \text {; calafrios } \\
(66,7 \%), \text { suores noturnos } \\
(33,3 \%) \text {, taquicardia }(33,3 \%) \text { e } \\
\text { taquipneia }(66,7 \%) .\end{array}$ & $\begin{array}{l}\text { Febre }(50,1 \%), \text { tosse } \\
\text { seca }(66,8 \%), \text { tosse } \\
\text { produtiva }(16,7 \%), \\
\text { mialgia }(33,4 \%), \\
\text { fadiga }(16,7 \%), \\
\text { cefaleia }(16,7 \%) \text { e } \\
\text { perda de peso aparente } \\
(83,5 \%) .\end{array}$ & $\begin{array}{l}\text { Febre e dor } \\
\text { muscular. }\end{array}$ & $\begin{array}{l}\text { Tontura, } \\
\text { cefaleia, } \\
\text { vômitos. }\end{array}$ & $\begin{array}{l}\text { Tosse produtiva }(50 \%) \\
\text { ou não produtiva } \\
(50 \%), \quad \text { dispneia } \\
(\text { SatO2: } 87 \%) \text { diarreia } \\
(50 \%) .\end{array}$ & $\begin{array}{lr}\text { Febre } & (62,5 \%), \\
\text { Dispneia } & (25 \%) \text {, Tosse } \\
(37,5 \%) \text { e Vômito } \\
(12,5 \%) .\end{array}$ \\
\hline $\begin{array}{l}\text { Exames } \\
\text { laboratoriais }\end{array}$ & $\begin{array}{l}\text { RT-PCR do swab } \\
\text { nasofaríngeo positivo } \\
\text { para SARS-CoV-2. PCR } \\
\text { para SARS-CoV-2 com } \\
\text { líquido pleural negativo } \\
(75 \%) \text { ou não realizado } \\
(25 \%) \text { IGRA positivo } \\
\text { para TB }(100 \%) \text {; } \\
\text { esfregaço de escarro } \\
\text { negativo para BAAR } \\
(100 \%) ; \text { cultura de } \\
\text { escarro para BAAR } \\
\text { pendentes (50\%) ou } \\
\text { negativas }(50 \%) \text { cultura } \\
\text { de líquido pleural para } \\
\text { BAAR pendente }(50 \%), \\
\text { não realizada }(25 \%) \text { ou } \\
\text { positiva }(25 \%) .\end{array}$ & $\begin{array}{l}\text { RNA de SARS- } \\
\text { CoV-2 positivo } \\
\text { em swab } \\
\text { nasofaríngeo e } \\
\text { LCR, IgA e IgG } \\
\text { consistente com } \\
\text { infecção aguda. } \\
\text { Leucócitos } 10,8 \\
\text { bil/L (linfócitos } \\
1,8 \mathrm{bil} / \mathrm{L}) \text {, VHS } \\
30 \mathrm{~mm} / \mathrm{h}, \text { sódio } \\
133 \mathrm{mmol} / \mathrm{L} \text {. }\end{array}$ & $\begin{array}{l}\text { Esfregaço de escarro positivo } \\
\text { para BAAR }(66,7 \%) \text { e M. } \\
\text { tubercuosis }(33,3 \%) \text {. RT-PCR } \\
\text { do swab nasofaríngeo positivo } \\
\text { para SARS-CoV-2 }(66,7 \%) \text { ou } \\
\text { não realizado }(33,3 \%) \\
\text { Leucocitose, baixa contagem de } \\
\text { células T, trombocitose, } \\
\text { elevação de TP, PTTa, INR, } \\
\text { ALT, AST ou LDH (33,3\%). } \\
\text { Queda de AST, albumina ou } \\
\text { creatinina (33,3\%). Neutrofilia, } \\
\text { linfopenia, baixa contagem de } \\
\text { CD4, queda de hemoglobina, e } \\
\text { elevação de D-dímero, } \\
\text { bicarbonato e/ou proteína C } \\
\text { reativa em } 2 \text { pacientes cada. } \\
\text { Elevação de fibrinogênio e } \\
\text { VHS (100\%). }\end{array}$ & 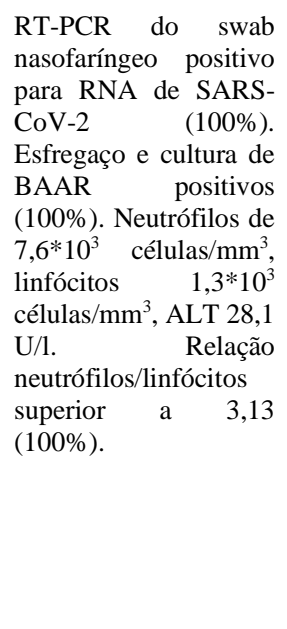 & $\begin{array}{l}\text { RT-PCR do swab } \\
\text { nasofaríngeo } \\
\text { indeterminado para } \\
\text { SARS-CoV-2. RT- } \\
\text { PCR e cultura de } \\
\text { Bacillus de Koch } \\
\text { (KB) negativos. } \\
\text { Linfopenia e } \\
\text { aumento de proteína } \\
\text { C reativa, } \\
\text { procalcitonina, } \\
\text { fibrinogênio e D- } \\
\text { dímero. }\end{array}$ & $\begin{array}{l}\text { RT-PCR de } \\
\text { swab } \\
\text { nasofaríngeo } \\
\text { positivo para } \\
\text { SARS-CoV-2 } \\
\text { e teste } \\
\text { QuantiFERO } \\
\mathrm{N} \text { positivo } \\
\text { para TB. } \\
\text { Linfopenia } \\
\text { com } \\
\text { contagem } \\
\text { normal de } \\
\text { leucócitos; } \\
\text { função renal e } \\
\text { hepática } \\
\text { normais. }\end{array}$ & $\begin{array}{l}\text { RT-PCR do swab } \\
\text { nasofaríngeo negativo } \\
\text { para SARS-CoV-2 } \\
(50 \%) \text { ou não realizado } \\
(50 \%) \text { Linfopenia, } \\
\text { níveis elevados de } \\
\text { proteína C reativa e } \\
\text { LDH. }\end{array}$ & $\begin{array}{l}\text { RT-PCR positivo para } \\
\text { SARS-CoV-2. Cultura } \\
\text { e/ou microscopia } \\
\text { direta positivas para } \\
\text { TB. }\end{array}$ \\
\hline
\end{tabular}


Research, Society and Development, v. 10, n. 2, e0710212257, 2021

(CC BY 4.0) | ISSN 2525-3409 | DOI: http://dx.doi.org/10.33448/rsd-v10i2.12257

\begin{tabular}{|c|c|c|c|c|c|c|c|c|}
\hline Autores & THAM et al., 2020 & $\begin{array}{l}\text { FREIJ et al., } \\
2020\end{array}$ & YAO et al., 2020 & YOUSAF et al., 2020 & $\begin{array}{l}\text { LUCIANI et al., } \\
2020\end{array}$ & $\begin{array}{l}\text { ATA et al., } \\
2020\end{array}$ & $\begin{array}{l}\text { SARINOGLU et al., } \\
2020\end{array}$ & MOTTA et al., 2020 \\
\hline $\begin{array}{ll}\text { Exames } \\
\text { imagem }\end{array}$ & $\begin{array}{l}\text { RX de tórax com } \\
\text { cavitações em região } \\
\text { superior direita e inferior } \\
\text { esquerda }(25 \%) \text {, derrame } \\
\text { pleural unilateral }(75 \%) \text { e } \\
\text { atelectasias }(25 \%) \text {. TC } \\
\text { de tórax com } \\
\text { opacificações irregulares } \\
\text { e cavitação central } \\
(25 \%) \text { derrame com } \\
\text { colapso/consolidação } \\
(50 \%) \text { e vidro fosco com } \\
\text { espessamento do septo } \\
\text { lobar }(25 \%) \text {. }\end{array}$ & Não realizado. & $\begin{array}{l}\text { TC de tórax revelou } \\
\text { opacificação em vidro fosco } \\
\text { nos } 3 \text { pacientes e derrame } \\
\text { pleural em dois deles. }\end{array}$ & $\begin{array}{l}\text { RX de tórax com } \\
\text { cavitações } \quad(100 \%) \text {. } \\
\text { Derrame pleural } \\
\text { exsudativo linfocítico } \\
(16,7 \%) \text { positivo para } \\
\text { TB. }\end{array}$ & $\begin{array}{l}\text { TC de tórax com } \\
\text { áreas de } \\
\text { consolidação } \\
\text { bilateralmente; } \\
\text { derrame pleural à } \\
\text { direita; atelectasia } \\
\text { do lobo inferior } \\
\text { direito. }\end{array}$ & $\begin{array}{l}\mathrm{RX} \text { de tórax } \\
\text { com } \\
\text { opacidades } \\
\text { bilaterais } \\
\text { irregulares. }\end{array}$ & $\begin{array}{l}\text { TC com perda do } \\
\text { volume no lobo } \\
\text { superior evoluindo } \\
\text { para aspecto de } \\
\text { "árvore } \\
\text { brotamento" em lobo } \\
\text { direito }(50 \%) \text {. Derrame } \\
\text { pleural e pericárdico } \\
(50 \%) ; \text { linfadenopatia } \\
\text { mediastinal }(50 \%) ; \\
\text { pneumonia rom } \\
\text { calcificações em lobo } \\
\text { superior }(50 \%) \text {; vidro } \\
\text { fosco (50\%); lesões } \\
\text { nodulares bilaterais } \\
(50 \%) .\end{array}$ & $\begin{array}{lrr}\text { Lesões bilaterais } & \mathrm{em} \\
98,6 \% & (6), \text { desses } & 2 \\
\text { com padrão miliar, } 1 & 1 \\
\text { pavimentação } & \mathrm{em} \\
\text { mosaico e } 1 & \mathrm{com} \\
\text { padrão árvore } & \mathrm{em} \\
\text { brotamento. } & \end{array}$ \\
\hline Evolução & $\begin{array}{l}\text { Todos foram tratados } \\
\text { com terapia anti-TB e } \\
\text { apresentaram resoluçãa } \\
\text { dos sintomas com } \\
\text { melhora no RX de tórax. }\end{array}$ & 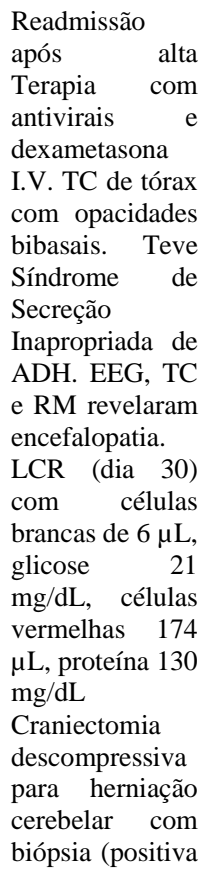 & $\begin{array}{l}\text { Todos tratados antibióticos e } \\
\text { antivirais. Dois pacientes } \\
\text { tratados com terapia anti-TB. } \\
\text { Intereron alfa usado em um } \\
\text { paciente, bem como } \\
\text { imunoglobulinas. Dois } \\
\text { precisaram de corticoides e e } \\
\text { suporte de oxigênio (estado } \\
\text { severo). A duração da } \\
\text { hospitalização foi de } 22 \text { a } 27 \\
\text { dias e o tempo entre o início } \\
\text { dos sintomas e o desfecho } \\
\text { variou de } 35 \text { a } 46 \text { dias. Um } \\
\text { paciente só foi diagnosticado } \\
\text { com COVID-19 após óbito } \\
\text { (RT-PCR do swab nasofaríngeo } \\
\text { positivo para SARS-CoV-2) }\end{array}$ & $\begin{array}{l}\text { Todos tratados com } \\
\text { ceftriaxona, } \\
\text { azitromicina } \\
\text { hidroxicloroquina para } \\
\text { pneumonia } \\
\text { COVID-19 por } \\
\text { dias), além de terapia } \\
\text { anti-TB. Todos } \\
\text { responderam bem ao } \\
\text { tratamento. }\end{array}$ & $\begin{array}{l}\text { Antibioticoterapia } \\
\text { (claritromicina }+ \\
\text { piperacilina/taobact } \\
\text { am) e tratamento } \\
\text { para COVID-19 } \\
\text { (hidroxicloroquina } \\
+ \\
\text { lopinavir/ritonavir). } \\
\text { RT-PCR do swab } \\
\text { nasofaríngeo } \\
\text { positivo para } \\
\text { SARS-CoV-2 no } 4^{\circ} \\
\text { dia de internação. } \\
\text { Antibióticos em uso } \\
\text { substituídos por } \\
\text { linezolida (7º dia) e } \\
\text { realizada } \\
\text { toracocentese } \\
\text { (cultura negativa } \\
\text { para KB). Recidiva } \\
\text { do derrame. Biópsia } \\
\text { pulmonar positiva } \\
\text { para KB). }\end{array}$ & $\begin{array}{l}\text { Tratado com } \\
\text { azitromicina e } \\
\text { hidroxicloroq } \\
\text { uina. } \\
\text { Craniotomia } \\
\text { suboccipital e } \\
\text { ressecção para } \\
\text { massa } \\
\text { cerebelar. TC } \\
\text { de tórax com } \\
\text { vidro fosco } \\
\text { bilateral e } \\
\text { sombra } \\
\text { reticulonodula } \\
\text { r, } \\
\text { espessamento } \\
\text { apical à } \\
\text { direita, } \\
\text { centrolobular } \\
\text { difuso e septal } \\
\text { peribrônquico } \\
\text { abc de } \\
\text { abdome com } \\
\text { lesões } \\
\text { hepáticas (TB } \\
\text { disseminada). } \\
\text { Biópsia e }\end{array}$ & 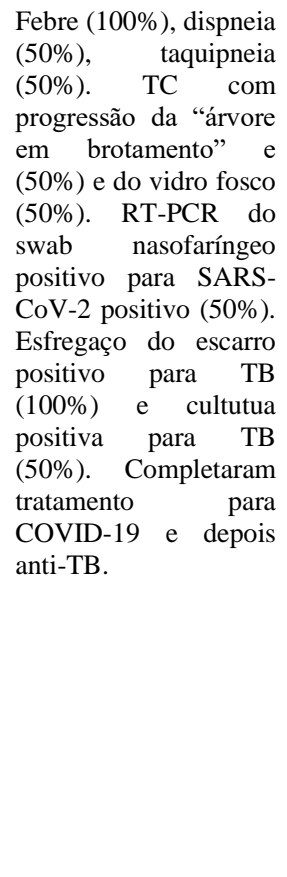 & $\begin{array}{l}\text { Tratados com terapia } \\
\text { anti-TB } \quad(100 \%), \\
\text { hidroxicloroquina } \\
(62,5 \%) \text { e antivirais } \\
(25 \%) \text {. } 4 \text { (50\%) } \\
\text { necessitaram r de } \\
\text { oxigenoterapia por } \\
\text { meio de máscara facial } \\
\text { e } 1 \text { foi submetido à } \\
\text { ventilaçãa r não } \\
\text { invasiva. A } \\
\text { média (intervalo) de } \\
\text { internaçãa hospitalar } \\
\text { foi de 27,5 (8-130) } \\
\text { dias. }\end{array}$ \\
\hline
\end{tabular}


Research, Society and Development, v. 10, n. 2, e0710212257, 2021

(CC BY 4.0) | ISSN 2525-3409 | DOI: http://dx.doi.org/10.33448/rsd-v10i2.12257

\begin{tabular}{|c|c|c|c|c|c|c|c|c|}
\hline & & $\begin{array}{l}\text { para SARS- } \\
\text { CoV-2, BAAR e } \\
\text { DNA de } \\
\text { Mycobacterium } \\
\text { tuberculosis). }\end{array}$ & & & & $\begin{array}{l}\text { PCR } \\
\text { confirmaram } \\
\text { TB pulmonar } \\
\text { envolvendo } \\
\text { SNC. } \\
\text { Instituída } \\
\text { terapia anti- } \\
\text { TB com } \\
\text { esteróides. } \\
\text { Assintomático } \\
10 \text { dias após } \\
\text { cirurgia. }\end{array}$ & & \\
\hline Desfecho & Não informado. & Óbito. & $\begin{array}{l}\text { Alta }(66,7 \%) \text { ou óbito por } \\
\text { falência cardiorrespiratória } \\
(33,3 \%) .\end{array}$ & Alta (100\%). & $\begin{array}{lr}\text { Alta } & \text { com } \\
\text { seguimento } & \text { de } \\
\text { terapia anti-TB. } & \end{array}$ & $\begin{array}{l}\text { Alta com } 28 \\
\text { dias de } \\
\text { internação. } \\
\text { Seguimento } \\
\text { de terapia } \\
\text { anti-TB. }\end{array}$ & Não informado. & $\begin{array}{l}8 \quad(11,6 \%) \text { de } 69 \\
\text { morreram após média } \\
\text { de } 9 \text { dias (variação 6- } \\
\text { 14) do diagnóstico de } \\
\text { COVID. }\end{array}$ \\
\hline
\end{tabular}

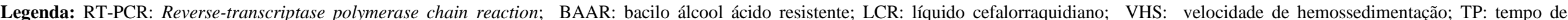

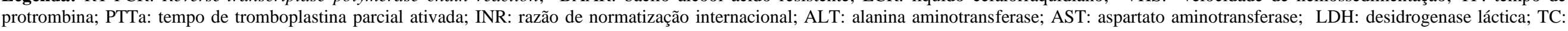
tomografia computadorizada; RX: raio X; RM: ressonância magnética; EEG: eletroencefalograma; I.V.: intravenosa; ADH: hormônio antidiurético. Fonte: Autores. 


\section{Discussão}

A infecção pelo SARS-CoV-2 passou a ser considerada uma pandemia pela OMS em 11 de março de 2020. Diante desse paradigma, profissionais de saúde em todo o mundo estudam a nova doença e sua interação com outras patologias. Nesse contexto, a coinfecção da COVID-19 com a tuberculose (TB) merece atenção especial, tanto pela elevada morbidade ocasionada pela tuberculose pulmonar, quanto pela alta prevalência da doença no Brasil, país com maior número de pacientes com TB das Américas (Ministério da Saúde, 2017).

Com relação ao perfil demográfico, no presente estudo, a maioria dos artigos incluídos (87,5\%) avaliou apenas um gênero, não estabelecendo, portanto, prevalência de coinfecção por sexo. Em um dos estudos avaliados (Motta et al. 2020), todavia, a coinfecção mostrou-se maior em indivíduos do sexo masculino (87,5\%). A literatura aponta que a maior parte dos indivíduos infectados pelo SARS-CoV-2 é do sexo masculino (Teich et al. 2020).

No que tange à idade, a média dos pacientes avaliados com coinfecção foi de 47,19 anos. Esse valor é superior à média de idade dos pacientes infectados por COVID-19 isoladamente no Brasil, que é de 39,9 anos (Teich et al. 2020). A correlação entre tuberculose e gravidade da COVID-19 não parece ser afetada pela média de idade dos pacientes coinfectados (Gao et al. 2020). Em contrapartida, a relação entre idade avançada e severidade da COVID-19 isolada já foi bem descrita na literatura, incluindo aumento dos óbitos intra-hospitalares pela doença em indivíduos mais idosos (Yang \& Lu, 2020).

Quanto aos sintomas mais frequentes (febre, tosse, dispneia e cefaleia), verifica-se que não há alteração dos achados para aqueles já esperados nos pacientes com COVID-19 sem a coinfecção (Ministério da Saúde, 2020). Entretanto, Motta et al. (2020) cita que em um número menor de casos pode haver secreção purulenta associada a tosse e a febre pode ser arrastada, achados menos frequentes em casos de SARS-CoV-2 isolados, mas que podem chamar a atenção do médico para o duplo diagnóstico.

No presente estudo, os principais achados laboratoriais foram linfopenia e elevação de proteína $\mathrm{C}$ reativa, velocidade de hemossedimentação, D-dímero e fibrinogênio. Conforme as Diretrizes para Diagnóstico e Tratamento da COVID-19 no Brasil, as principais anormalidades laboratoriais em casos graves de infecção pelo SARS-CoV-2 são leucopenia, linfopenia, leucocitose e transaminases hepáticas elevadas, além de neutrofilia, trombocitopenia e elevação de creatinina sérica. Ademais, nesses casos níveis séricos elevados de ALT, lactato desidrogenase (DHL), troponina I cardíaca de alta sensibilidade, creatina quinase, D-dímero, ferritina sérica, IL-6, creatinina, procalcitonina e tempo de protrombina são preditores de letalidade (Ministério da Saúde, 2020).

Como a TB tem início insidioso, ela geralmente está presente antes da COVID-19, mas devido ao subdiagnóstico de TB e à ampla divulgação da pandemia, o diagnóstico da COVID-19 tem acontecido antes da TB (Khurama \& Aggarwal, 2020). Entretanto, em Motta et al. (2020) 75\% dos pacientes foram diagnosticados para COVID-19 de 7 a 75 dias (média: 22,5 dias) após o diagnóstico de TB.

Quando solicitados exames de imagem, o principal método utilizado foi a tomografia computadorizada (TC) de tórax que mostrou nos pacientes uma sobreposição geralmente bilateral de achados de ambas as condições, como a árvore em brotamento, cavidades ou consolidações da tuberculose com o padrão em vidro fosco ou espessamento intersticial do COVID19 (Luciani et al. 2020; Motta et al. 2020; Sarinoglu et al. 2020; Tham, et al. 2020; Yao et al. 2020). O raio X (RX) de tórax também apresentou achados de ambas as patologias, como cavitações, opacificações e derrame pleural (Ata et al. 2020; Sarinoglu et al. 2020; Tham, et al. 2020; Yousaf et al. 2020). Nenhum dos trabalhos analisou se o padrão de imagem da TC ou do RX de tórax dos pacientes coinfectados era mais grave que o de pacientes apenas com COVID-19.

Muitas medicações amplamente utilizadas nos estudos avaliados não estão em consonância com as diretrizes pactuadas pela Associação de Medicina Intensiva Brasileira, Sociedade Brasileira de Infectologia e Sociedade Brasileira de Pneumologia e Tisiologia em maio de 2020 (publicação em julho de 2020), para as quais não há indicação para uso de rotina 
de hidroxicloroquina, cloroquina, azitromicina, lopinavir/ritonavir, corticosteroides ou tocilizumabe no tratamento da COVID19 (Falavigna et al. 2020). Ainda de acordo com Falavigna et al. (2020), os antibacterianos só devem ser considerados nos pacientes com suspeita de coinfecção bacteriana, condizente com o apresentado nos relatos. Entretanto, como referido por Luciani et al. (2020), antibióticos, imunossupressores, heparina e drogas antivirais têm sido largamente utilizadas para tratamento da COVID-19 atualmente em diversos países. Nesse sentido, Romeral, Caldeira, Alves-Olher e Vandresen (2020) destacam que muitos fármacos demonstraram efeito positivo contra o SARS-CoV-2 em resultados preliminares de ensaios clínicos realizados com pequenos grupos infectados e atualmente, ensaios controlados randomizados e potenciais vacinas estão desenvolvimento.

Dois autores relataram complicações neurológicas em pacientes coinfectados (Ata et al. 2020; Freij et al. 2020) que demandaram intervenção neurocirúrgica. A literatura aponta que os coronavírus apresentam potencial neuroinvasivo e mesmo na ausência de coinfecção por TB podem cursar com sintomas neurológicos como cefaleia, tontura, hiposmia e acidente vascular encefálico isquêmico ou hemorrágico (Freij et al., 2020).

A duração da hospitalização dos pacientes no estudo de Yao et al. (2020) foi de 22 a 27 dias, similar ao encontrado por Ata et al. (2020) que foi de 28 dias e Grenho et al. (2020), que foi de 10 dias em análise retrospectiva de pacientes internados infectados pelo SARS-CoV-2 com outras morbidades exceto a TB. Quanto maior o tempo de internamento maior o risco de iatrogenias e infecções secundárias, o que pode levar a um aumento da letalidade da doença nesses pacientes. Quando a coinfecção foi analisada diante da gravidade da pneumonia por COVID-19 notou-se que está muito mais associada a casos graves da doença (78\%) do que a casos leves a moderados (22\%) (Maciel, Gonçalves Júnior \& Dalcolmo, 2020).

O mecanismo exato de interação da COVID-19 e TB ainda é desconhecido. Os interferons tipo I (ex.: IFN- $\alpha$, IFN- $\beta$ ) são ativados precocemente em uma infecção viral e são fundamentais na imunidade antiviral (Freij et al. 2020). Entretanto, múltiplos estudos demonstraram que essa resposta pode comprometer a capacidade macrofágica de conter o crescimento micobacteriano, incluindo da Mycobacteruim tuberculosis (Freij et al. 2020). Sendo assim, a resposta imune à COVID-19 pode aumentar a suscetibilidade à infecção por TB quando promover uma resposta por interferon tipo I inadequada ou mal programada (Yousaf et al. 2020). Também acredita-se que possa haver uma ação combinada de diversas interleucinas ativadas pela resposta inflamatória viral que afeta a resposta imune de células T (Yousaf et al. 2020). Não obstante, é conhecido que o status imune vulnerável torna o indivíduo suscetível às duas patologias em questão (Sarinoglu et al. 2020).

Os trabalhos divergiram quanto à letalidade e à real importância da tuberculose como fator de risco para a infecção por coronavírus. Os oito pacientes avaliados por Motta et al. (2020) faziam parte de um estudo maior composto por 69 pacientes e sucumbiram à pneumonia por SARS-CoV-2 (letalidade de 11,6\%). No estudo de Yao et al. (2020) um (33,3\%) dos três pacientes foi a óbito e no relato de Freij et al. (2020) o paciente também faleceu por complicações cardiovasculares e neurológicas, respectivamente. O estudo de Tadolini et al. (2020a), que também investigou a coinfecção por COVID-19 e TB, identificou que 6 dos 49 (12,3\%) foram a óbito, resultado similar a Motta et al. (2020). Um fato digno de nota é que diferentemente de outros casos relatados na literatura (Stochino et al. 2020; Tadolini et al. 2020a), o paciente de Yao et al. (2020) que foi a óbito não possuía outras comorbidades sabidamente reconhecidas como fatores de risco (idade > 65 anos, Diabetes Melitus, Obesidade). Para Motta et al. (2020), nos casos em que há condições prévias de risco é possível que os óbitos se devam à associação entre a infecção do SARS-CoV-2 e os outros fatores e, portanto, a tuberculose não deve ser considerada como o fator de risco determinante para a letalidade nesses casos.

Para além das consequências clínicas dessa coinfecção, há também interferências sociais. Segundo Guimarães, Lobo, Siqueira, Borges e Melo (2012) há forte associação bidirecional entre a tuberculose e a pobreza havendo uma maior prevalência da doença em países subdesenvolvidos da América Latina, África e Ásia. Tham et al. (2020) e Yousaf et al. (2020) reforçam essa afirmação ao considerarem que os fatores de risco para TB incluem baixo nível socioeconômico e condições de 
vida superlotadas. Em seu estudo com trabalhadores migrantes, Tham et al. (2020) notou que os dormitórios costumam ser inadequadamente ventilados e lotados, o que torna os residentes mais suscetíveis a doenças infecciosas, incluindo dengue, zika, varicela, COVID-19 e TB.

Além disso, Kasaeva (diretora do programa global de tuberculose) afirma que a pandemia de COVID-19 pode afetar os pacientes com TB indiretamente, através das medidas de restrição, cancelamentos de voos e suspensão do transporte de cargas através das fronteiras ou dentro dos territórios, as quais comprometem a entrega das medicações para o tratamento do bacilo de Koch (Adepoju, 2020). Esse impacto acentua-se em países pobres diante da alta prevalência da TB, das dificuldades logísticas e da maior dependência da importação desses fármacos.

Boffa et al. (2020) reflete sobre o fato de o auto isolamento ser um desafio a mais para o diagnóstico dos pacientes com TB, pois estes já sofrem com a marginalização, o que demanda fortalecimento das políticas de busca ativa desses pacientes.

Apesar dos resultados apresentados, o presente estudo possui limitações. Estas estão principalmente associadas ao pequeno número de trabalhos publicados acerca do tema, representados, em sua maioria, por relatos de caso, e da variabilidade no modelo de apresentação das informações entre os estudos. Também são fatores limitantes o pequeno número amostral dos trabalhos disponíveis, havendo, portanto, uma possibilidade significativa de os resultados obtidos não se replicarem em estudos maiores. Diante do grande impacto da COVID-19 e suas repercussões no dia-a-dia do clínico, bem como considerando as complicações associadas à tuberculose, é de suma importância que novas pesquisas científicas sejam realizadas sobre o tema, a fim de direcionar as ações e protocolos de saúde.

\section{Conclusão}

Não se pode afirmar que a TB aumenta a taxa de mortalidade nos pacientes infectados por SARS-CoV-2, visto que a maioria dos pacientes que foram a óbito tinham fatores de mau prognóstico já bem apontados na literatura, como idade $>60$ anos e comorbidades. No entanto, observou-se uma tendência dos pacientes coinfectados a evoluírem com quadro moderado ou grave, incluindo necessidade de hospitalização, o que aponta para influência do estado imunológico do paciente com tuberculose na COVID-19.

Entretanto, visto que a população dos artigos incluídos era pequena, novos estudos fazem-se necessários para elucidar a temática. Nesse sentido, o estabelecimento de colaboração internacional em pesquisa é vital para conduzir estudos de forma rápida e coordenada. Ademais, dados epidemiológicos usando desenhos de coorte ou ensaios clínicos randomizados são necessários para estabelecer a influência mútua entre COVID-19 e tuberculose. As investigações laboratoriais sobre os mecanismos fisiopatológicos e imunológicos da doença demandam futuros estudos para desenvolvimento de manejo e tratamento adequados.

Por fim, é fundamental que os profissionais e serviços de saúde estejam atualizados e capacitados a realizarem diagnóstico diferencial entre ambas as patologias com vistas a melhoria de prognóstico.

\section{Referências}

Adepoju, P. (2020). Tuberculosis and HIV responses threatened by COVID-19. The Lancet HIV, 7 (5), e319-e320.

Ata, F., Yousaf, Q., Parambil, J. V., Parengal, J., Mohamedali, M. G., \& Yousaf, Z. (2020). A 28-Year-Old Man from India with SARS-Cov-2 and Pulmonary Tuberculosis Co-Infection with Central Nervous System Involvement. The American journal of case reports, 21, e926034-1-e926034-5.

Bandyopadhyay, A., Palepu, S., Bandyopadhyay, K., \& Handu, S. (2020). COVID-19 and tuberculosis co-infection: a neglected paradigm. Monaldi Archives for Chest Disease, 90 (3), 518-522.

Boffa, J., Mhlaba, T., Sulis, G., Moyo, S., Sifumba, Z., Pai, M., \& Daftary, A. (2020). COVID-19 and tuberculosis in South Africa: A dangerous combination. SAMJ: South African Medical Journal, 110 (5), 1-2. 
Chen, Y., Wang, Y., Fleming, J., Yu, Y., Gu, Y., Liu, C., Fan, L., Xiaodan, W., Cheng, M., Bi, L., \& Liu, Y. (2020). Active or latent tuberculosis increases susceptibility to COVID-19 and disease severity. MedRxiv. https://doi.org/10.1101/2020.03.10.20033795.

Falavigna, M., Colpani, V., Stein, C., Azevedo, L. C. P., Bagattini, A. M., Brito, G. V. de, Chatkin J. M., Cimerman, S., Corradi, N. de F. D. B., Cunha, C. A. da, Medeiros, F. C. de, Oliveira Junior, H. A. de, Fritscher, L. G., Gazzana, M. B., Gräf1, D. D., Marra, L. P., Matuoka, J. Y., Nunes, M. S., Pachito, D. V., Pagano1, C. G. M., Parreira, P. do C. S., Riera, R., Silva Júnior, A., Tavares, B. de M., Zavascki, A. P., Rosa1, R. G., \& Dal-Pizzol, F. (2020). Diretrizes para o tratamento farmacológico da COVID-19. Consenso da Associação de Medicina Intensiva Brasileira, da Sociedade Brasileira de Infectologia e da Sociedade Brasileira de Pneumologia e Tisiologia. Revista Brasileira de Terapia Intensiva, 32 (2), 166-196.

Freij, B. J., Gebara, B. M., Tariq, R., Wang, A. M., Gibson, J., El-Wiher, N., Krasan, G., Patek, P. M., Levasseur, K. A., Amin, M., \& Fullmer, J. M. (2020). Fatal Central Nervous System Co-Infection with SARS-CoV-2 and Tuberculosis in a Healthy Child. BMC Pediatrics, 20 (1), 429.

Gao, Y., Liu, M., Chen, Y., Shi, S., Geng, J., \& Tian, J. (2020). Association between tuberculosis and COVID-19 severity and mortality: A rapid systematic review and meta-analysis. Journal of Medical Virology. 10.1002/jmv.26311

Grenho, F., Lourenço, A., Ventura, S., Bogalho, I., Bargiela, I., Silva, C. S., Martins, F., Hatchbach, L., Lopes, N., Prades, A., \& Gomes, M. J. (2020). Características Clínicas de Doentes com COVID-19 num Internamento Dedicado do Hospital CUF Infante Santo: Série de Casos. Gazeta Médica, 7 (2), 82-91.

Guimarães, R. M., Lobo, A. D. P., Siqueira, E. A., Borges, T. F. F., \& Melo, S. C. C. (2012). Tuberculose, HIV e pobreza: tendência temporal no Brasil, Américas e mundo. Jornal brasileiro de Pneumologia, 38 (4), 511-517.

He, G., Wu, J., Shi, J., Dai, J., Gamber, M., Jiang, X., Sun, W., \& Cai, J. (2020). COVID-19 in Tuberculosis patients: a report of three cases. Journal of Medical Virology, 92 (10), 1802-1806.

Khurana, A. K., \& Aggarwal, D. (2020). The (in) significance of TB and COVID-19 co-infection. European Respiratory Journal, 56 (2), $56-58$.

Köche, J. C. (2011). Fundamentos de metodologia científica. (Edição Digital). Rio de Janeiro: Editora Vozes.

Kumar, R., Bhattacharya, B., Meena, V., Soneja, M., \& Wig, N. (2020). COVID-19 and TB co-infection-'Finishing touch"in perfect recipe to'severity'or 'death'. Journal of Infection, 81 (3), e39-e40.

Lai, C. C., \& Yu, W. L. (2020). The COVID-19 Pandemic and Tuberculosis in Taiwan. The Journal of Infection, 81 (2), e159-e161.

Luciani, M., Bentivegna, E., Spuntarelli, V., Lamberti, P. A., Guerritore, L., Chiappino, D., Nalli, G., Proietta, M., Porto, F. D., Martelletti P., \& Sesti, G. (2020). Coinfection of Tuberculosis Pneumonia and COVID-19 in a Patient Vaccinated with Bacille Calmette-Guérin (BCG): Case Report. SN comprehensive clinical medicine, 2 (11), 2419-2422.

Lüdke, M., \& André, M. (2013). Pesquisa em educação: abordagens qualitativas (2a ed.). E.P.U.

Maciel, E. L. N., Gonçalves Júnior, E., \& Dalcolmo, M. M. P. (2020). Tuberculose e coronavírus: o que sabemos? Epidemiologia e Serviços de Saúde, 29 (2), e2020128.

Ministério da Saúde. (2017). Brasil Livre da Tuberculose: Plano Nacional pelo Fim da Tuberculose como Problema de Saúde Pública. http://bvsms.saude.gov.br/bvs/publicacoes/brasil_livre_tuberculose_plano_nacional.pdf.

Ministério da Saúde. (2020). Diretrizes para Diagnóstico e Tratamento da COVID-19. https://portalarquivos.saude.gov.br/images/pdf/2020/May/13/DiretrizCovid19-v4.pdf

Motta, I., Centis, R., D’Ambrosio, L., García-García, J. M., Goletti, D., Gualano, G., Lipani, F., Palmieri, F., Sánchez-Montalvá, A., Pontali, E., Sotgiu, G., Spanevello, A., Stochino, C., Tabernero, E., Tadolini, M., van den Boom, M., Villa, S., Visca, D., \& Migliori, G. B. (2020). Tuberculosis, COVID-19 and migrants: preliminary analysis of deaths occurring in 69 patients from two cohorts. Pulmonology, 26 (4), 233-240.

Pereira, A. S., Shitsuka, D. M., Parreira, F. J., \& Shitsuka, R. (2018). Metodologia da pesquisa científica.[e-book]. Santa Maria. Ed (pp. 3-9). UAB/NTE/UFSM. https://repositorio. ufsm. br/bitstream/handle/1/15824/Lic_Computacao_Metodologia-Pesquisa-Cientifica. pdf.

Romeral, R. D. A. F., Caldeira, M. E. B., Alves-Olher, V. G., \& Vandresen, F. (2020). Repositioning drugs for treatment of SARS-CoV-2. Brazilian Journal of Development, 6 (11), 85263-85283.

Sarınoğlu, R. C., Sili, U., Eryuksel, E., Yildizeli, S. O., Cimsit, C., \& Yagci, A. K. (2020). Tuberculosis and COVID-19: An overlapping situation during pandemic. The Journal of Infection in Developing Countries, 14 (07), 721-725.

Singh, A., Prasad, R., Gupta, A., Das, K., \& Gupta, N. (2020). Severe acute respiratory syndrome coronavirus-2 and pulmonary tuberculosis: convergence can be fatal. Monaldi Archives for Chest Disease, 90 (3), 441-450.

Souza, M. T. D., Silva, M. D. D., \& Carvalho, R. D. (2010). Revisão integrativa: o que é e como fazer. Einstein, 8 (1), $102-106$.

Stochino, C., Villa, S., Zucchi, P., Parravicini, P., Gori, A., \& Raviglione, M. C. (2020). Clinical characteristics of COVID-19 and active tuberculosis coinfection in an Italian reference hospital. European Respiratory Journal, 56 (1), 2001708.

Tadolini, M., Codecasa, L. R., García-García, J. M., Blanc, F. X., Borisov, S., Alffenaar, J. W., Andréjak, C., Bachez, P., Bart, P. A., Belilovski, E., CardosoLandivar, J., Centis, R., D'Ambrosio, L., Souza-Galvão, M. L. de, Dominguez-Castellano, A., Dourmane, S., Jachym, M. F., Froissart, A., Giacomet, V., Goletti, D., Grard, S., Gualano, G., Izadifar, A., Du, D. L., Royo, M. M., Mazza-Stalder, J., Motta, I., Ong, C. W. M., Palmieri, F., Rivière, F., Rodrigo, T., Silva, D. R., Sánchez-Montalvá, A., Saporiti, M., Scarpellini, P., Schlemmer, F., Spanevello, A., Sumarokova, E., Tabernero, E., Tambyah, P. A., Tiberi, S., Torre, A., Visca, D., Murguiondo, M. Z., Sotgiu, G. \& Migliori, G. B. (2020). Active tuberculosis, sequelae and COVID-19 co-infection: first cohort of 49 cases. European Respiratory Journal, 56 (1), 2001398. 
Research, Society and Development, v. 10, n. 2, e0710212257, 2021

(CC BY 4.0) | ISSN 2525-3409 | DOI: http://dx.doi.org/10.33448/rsd-v10i2.12257

Tadolini, M., García-García, J. M., Blanc, F. X., Borisov, S., Goletti, D., Motta, I., Codecasa, L. R., Tiberi, S., Sotgiu, G., \& Migliori, G. B. (2020). On tuberculosis and COVID-19 co-infection. European Respiratory Journal, 56(2), 2002328.

Teich, V. D., Klajner, S., Almeida, F. A. S. D., Dantas, A. C. B., Laselva, C. R., Torritesi, M. G., Canero, T. R., Berwanger, O., Rizzo, L. V., Reis, E. P. \& Cendoroglo Neto, M. (2020). Características epidemiológicas e clínicas dos pacientes com COVID-19 no Brasil. Einstein (São Paulo), 18, 1-7.

Tham, S. M., Lim, W. Y., Lee, C. K., Loh, J., Premkumar, A., Yan, B., Kee, A., Chai, L., Tambyah, P. A., \& Yan, G. (2020). Four patients with COVID-19 and tuberculosis, Singapore, April-may 2020. Emerging Infectious Diseases, 26 (11), 2763-2765.

Yang, H., \& Lu, S. (2020). COVID-19 and tuberculosis. Journal of Translational Internal Medicine, 8 (2), 59-65.

Yao, Z., Chen, J., Wang, Q., Liu, W., Zhang, Q., Nan, J., Huang, H., Wu, Y., Li, L., Liang, L., You, L., Liu, Y., \& Yu, H. (2020). Three Patients with COVID19 and Pulmonary Tuberculosis, Wuhan, China, January-February 2020. Emerging infectious diseases, 26 (11), 2754.

Yousaf, Z., Khan, A. A., Chaudhary, H. A., Mushtaq, K., Parengal, J., Aboukamar, M., Khan, M. U., \& Mohamed, M. F. (2020). Cavitary pulmonary tuberculosis with COVID-19 coinfection. IDCases, 22, e00973. 